\title{
Überlegungen zu Formen und Funktionen der Ausrufesätze im Deutschen und Arabischen
}

\author{
Rasha Emadeldin Sayed Mohamed \\ Assistentin an der Abteilung für \\ Germanistik der Al-Alsun Fakultät der Minia Universität \\ rascha_nar@yahoo.com
}

\begin{abstract}
Abstrakt
Dieser vorliegende Beitrag beschäftigt sich mit dem Thema „Ausrufesätze" im Deutschen und Arabischen und setzt sich mit den Formen und Funktionen des deutschen und arabischen Ausrufesatzes auseinander. Darüber hinaus versucht der Beitrag diese Sätze zu untersuchen. Vor diesem Hintergrund behandelt dieser Beitrag die folgenden zentralen Fragestellungen:

1. welche Funktionen und Formen können die Ausrufesätze erfüllen?

2. wie unterscheiden sich die Ausrufesätze von den anderen Satzarten?

3. in wieweit stimmen solche Sätze in beiden Sprachen überein und in wieweit unterscheiden sie sich voneinander?
\end{abstract}

Der Beitrag übernimmt daher das kontrastive Verfahren. Damit setzt man sich zum Ziel, diese Art von Sätzen sowohl im Deutschen als auch im Arabischen zu konfrontieren.

Schlüsselwörter: Ausrufe, Ausrufe- bzw. Exklamativsätze, emotionale Funktion, Interjektion.

\section{Methodische Einleitung}

Man verwendet die Sprache nicht nur, um Gedanken $\mathrm{zu}$ formulieren oder mitzuteilen, sondern auch, um starke Emotionen und Gefühle wie Freude, Überraschung, Wut usw. auszudrücken. Diese emotionale Funktion erfüllen die Ausrufe- bzw. Exklamativsätze. Die Emotionalität steht in dieser Satzart sowohl im 
Deutschen als auch im Arabischen im Vordergrund (vgl. Rosengren, 1992: 264, vgl. Hassa:n, 2000: 138). In beiden Sprachen äußern die Ausrufesätze lebhafte Empfindungen und stellen emotionale Reaktionen auf ein Geschehen oder einen Sachverhalt dar.

In Anlehnung an die kontrastive Methode stellt diese Forschungsarbeit das Thema „Ausrufesätze" in zwei verschiedenen unverwandten Sprachen und zwar im Deutschen und im Arabischen dar. Dadurch werden die Gemeinsamkeiten und Unterschiede in beiden Sprachen vorgestellt. Der vorliegende Beitrag gliedert sich in drei Hauptteile. Der erste und zweite Teil widmen sich jeweils der terminologischen Klärung des Begriffs „Ausrufesatz" im Deutschen und Arabischen. Es wird eine Folge von Ausrufesätzen in verschiedenen Formen mit ihren spezifischen Strukturen in beiden Sprachen dargestellt. Der dritte Teil stellt den Vergleich zwischen den Ausrufesätzen im Deutschen und im Arabischen an und fasst die Schlussfolgerungen des Artikels zusammen.

\section{Der Ausrufesatz im Deutschen}

Im Deutschen unterscheiden viele Grammatiker wie Duden (1995) und Dürscheid (2012) fünf Satzarten und zwar Aussage-, Frage-, Aufforderungs-, Wunsch- und Ausrufesätze. Die Wahl der Satzart im Deutschen hängt von der Intention des Sprechers ab, eine Tatsache wiederzugeben oder etwas mitzuteilen, eine Frage oder einen Wunsch zu äußern, eine Aufforderung bzw. einen Befehl auszudrücken oder letztlich eine emotionale Einstellung und Beteiligung zum Ausdruck zu bringen (vgl. Dürscheid, 2012: 61/ Duden-Grammatik, 1995: 591).

\section{Beispiele $^{1}$ der Aussagesätze:}

- Paul war gestern nicht in der Schule.

- Paul behauptet, dass Lisa Egon liebt.

\section{Beispiele der Fragesätze:}


- Kommst du nicht mit ins Kino?

- Warum kommst du denn eigentlich nicht mit ins Kino?

\section{Beispiele der Aufforderungssätze:}

- Komm heute bloß nicht zu spät!

- Bitte fahren Sie langsam an der Unfallstelle vorbei!

\section{Beispiele der Wunschsätze:}

- Wenn sie nur bald käme!

- Wenn sie nur gekommen wäre!

\section{Beispiele der Ausrufe- bzw. Exklamativsätze:}

- Was du nicht sagst!

- Du bist mir aber ein netter Freund!

- Nun komm doch schon!

An dieser Stelle muss man besonders betonen, dass die Ausrufe- bzw. Exklamativsätze etwas mit Emotionalität zu tun haben; diesen Zusammenhang zwischen diesem Satztyp und dem Ausdruck von Emotionen hat Bussmann (2002) festgestellt, indem sie den Begriff „Exklamation“ im Allgemeinen definiert als „sprachlicher Handlungstyp (Illokutionstyp), der eine Einstellung des Erstaunens, des Überraschtseins, des Für-außergewöhnlich-Haltens gegenüber seinem propositionalen Gehalt ausdrückt“" (Bussmann, 2002: unter Exklamation 207).

Der Ausrufe- bzw. Exklamativsatz wird also durch die emotionale Einstellung des Sprechers charakterisiert. Die Exklamativsätze drücken laut Kürschner „ein Erstaunen oder eine andere psychische Regung” (Kürschner, 1989:257) aus. Unter „andere Gefühlsregungen“" versteht man beispielsweise begeisterte Zustimmung, Bewunderung oder Verwunderung, Widerwillen, Aufregung, Entsetzen, Gefühlskälte, Ärger, Wut, Freude, Spaß usw. Meistens äußern die Ausrufesätze die Gefühle der Bewunderung oder Verwunderung und bedeuten „Ich wundere mich, dass ..." oder „Ich bewundere“ (Kessel/Reimann, 2012: 7); wenn z.B. der Sprecher sagt: „Bist du aber gewachsen!“ bedeutet das „Ich wundere mich, wie sehr du 
gewachsen bist“". Dagegen beschränkt d'Avis (2013) den Begriff „Exklamationsatz“ auf den Ausdruck des Erstaunens über etwas Unerwartetes (vgl. d'Avis, 2013: 171); z.B. „Die ist aber schön!““ bedeutet: „Ich habe nicht erwartet, dass die so schön ist ${ }^{e e} \quad$ (http://www.karstenrinas.com/pdf/RinasPartikeln_als_Mumien.pdf, am 20/10/2016).

Neben den Bezeichnungen „Ausrufe- bzw. Exklamativsatz“ führen einige Grammatiker folgende Termini ein: „, Ausrufungssatz‘ (Paul 1968: 14, 41), ,intensivierende Ausrufe‘, ,w-Ausrufe‘, ,ist-das-Ausrufe‘ (Roncador 1977: 104)“ (d'Avis, 2013: 172).

Dürscheid charakterisiert die Ausrufesätze wie folgt:

Sie lassen sich in sytaktisch-formaler Hinsicht nur schwer eingrenzen. Das Verb kann sowohl in erster, zweiter oder letzter Position stehen, als Modus tritt sowohl der Indikativ als auch Konjunktiv II auf (Dürscheid, 2012: 64).

Die folgenden Beispiele zeigen, dass die Ausrufesätze im Deutschen verschiedene Formen mit verschiedenen formalen Merkmalen haben.

Die folgenden Beispiele sehen wie Aussagesätze aus²:

- Die ist (aber/vielleicht) schön!

- Du bist (aber/vielleicht) süß!

- Du bist (aber/vielleicht) groß geworden!

- Ich habe (aber/vielleicht) Hunger!

Die folgenden Beispiele sehen wie Fragesätze aus ${ }^{3}$ :

- Welchen TOLLEN Mann die aber auch geheiratet hat!

- Hat DIE aber ein schönes Fahrrad!

- Was DIE aber für große Füße hat!

Die folgenden Beispiele sehen wie Nebensätze aus :

- Dass DER die Josephine heiratet!

- Wie schnell der LÄUFT!

Wie die oben genannten Beispiele zeigen, sieht die Satzstruktur der Ausrufesätze meistens wie die Satzstruktur der Aussage- oder Fragesätze aus. Was aber die ersten 
vier Beispiele anbelangt, so macht der Sprecher keine Aussagen darüber, dass jemand bzw. etwas beispielsweise schön und nicht hässlich ist, sondern er äußert sein Erstaunen darüber. Der Unterschied zwischen Ausrufesätzen und Fragesätzen liegt darin, dass die Ausrufesätze keine Antwort erfordern; z.B.

- A: Wie groß ist sie? B: Ein Meter und achtzig.

- A: Wie GROSS die ist! B: \# Ein Meter und achtzig. (d'Avis, 2013: 191)

Zusätzlich liegt der Unterschied in dem Ausdruck der Gefühle, die zum Ausrufen führen (vgl. d'Avis, 2013: 175). Deshalb kommen meistens Partikeln wie aber oder vielleicht oder Interjektionen vor, die den Ausdruck der Emotion verstärken.

Die Ausrufesätze im Deutschen charakterisieren sich durch ihre fallende Intonation: „Diese Intonation drückt Emotionalität, vor allem Bewunderung oder Verwunderung aus.“ (Duden-Grammatik, 1995: 597).

\section{Der Ausrufesatz im Arabischen}

إنشاء غير Ausrufesätze wurden im Arabischen früher immer unter der Bezeichnung طلبى 'innša:' ġayr țalabi (wörtl. nicht auffordernder Ausruf - Exklamation) behandelt.

Der إنشاء غير طلبى ist eine Äußerung, die eine zum Zeitpunkt der Formulierung vorhandene mentale Einstellung oder eine Absicht offenbart, aber keine Forderung enthält. Es erfasst eine Vielzahl von Arten, wobei einige häufiger vorkommen. Manche davon entsprechen ebenfalls einem illokutiven Sprechakt (z.B. Lob) (Stock, 2004: 17).

In diesem Zusammenhang erwähnt Stock (2004) sechs Arten des إنشاء غير طلبى (Exklamation) und nämlich Eid, Tadel, Lob, Hoffnung, Erstaunen und Vertrag:

\begin{tabular}{|c|c|c|c|}
\hline Eid & قسم & $>$ Bei Gott, das tue ich nicht! & له والله لا أفعل هذا! \\
\hline Tadel & ذام & $>$ Diese Worte sind so übel[!] & 4 بئس هذا الكلام. \\
\hline Lob & مدح & $>$ Großartig ist diese Kunst[!] & \\
\hline Hoffnung & رجاء & $>$ Ach, vielleicht kommt er heute! & 4 \\
\hline Erstaunen & ت تعجب & $>$ Wie schön sie doch ist! & ما أجملها! \\
\hline Vertrag & عقد & $>$ Hiermit kaufe ich das Haus... & > إنتريت البيت... \\
\hline
\end{tabular}

Tabelle 1 Einige Arten des إنشاء غير طلبى (Stock, 2004: 17)

Hassa:n (1994) ist - meines Wissens - der Erste, der den arabischen Begriff الإفصاحيات 'alifṣa:hiyya:t (wörtl. die Expressiven) eingeführt hat, indem er den Satz in 


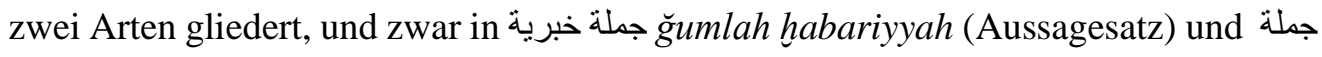
ğ إنشائية وumlah 'inša: 'iyah (performativer Satz), der sich entweder in جملة طلبية gumlah

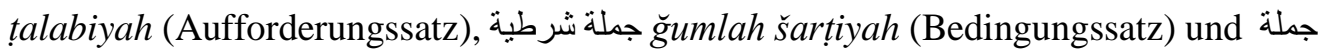
ğ gumlah 'ifșa:hiyyah (Ausrufesatz bzw. Exklamativsatz) einteilen lässt (vgl. Ḥassa:n, 1994: 244). Er bevorzugt إنشاء غير طلبى 'innša:' 'gayr țalabi (Exklamation) unter der Bezeichnung الجملة الإفصاحية أو التركيب الإفصاحى (Ausrufesatz bzw. Ausrufekonstruktion) zu behandeln, indem er feststellt:

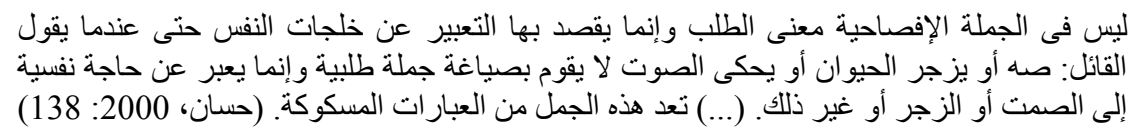

[Ü: Der Ausrufesatz enthält keine Forderung, sondern drückt innerliche Seelenregungen aus. Auch wenn der Sprecher șah (pst!, still!) sagt, das Tier antreibt oder den Laut imitiert, bildet er keinen Aufforderungssatz, sondern äußert einfach sein seelisches Bedürfnis zur Ruhe oder zur Zurechtweisung usw. (...) Diese Sätze zählen zu den festen Wortverbindungen.] (Hassa:n, 2000: 138). ${ }^{5}$

Darüber hinaus hat er die emotionale Funktion dieses Terminus festgestellt, indem er äußert:

$$
\begin{aligned}
& \text { "تستعمل للكثف عن موقف انفعالى ما والإفصاح عنه فهى من حيث استعمالها قريبة الثبة بما يسمونه فى } \\
& \text { اللغة الإنجليزية Exclamation" (حسان، 1994: 113) }
\end{aligned}
$$

[Ü: [sie] werden verwendet, um eine bestimmte emotionale Willenshaltung zu offenbaren oder auszudrücken. Bezüglich ihrer Verwendung sind sie dem englischen Begriff ,Exclamation` sehr ähnlich] (Hassa:n, 1994: 113).

Der emotionale Wert des Ausrufesatzes repräsentiert sich in dem Unterschied zwischen dem Ausruf (أوب!) 'awwah (oh!) und dem Aussagesatz (أتوجع) 'atawağa' (es tut mir weh), der erfragt werden kann (vgl. Ḥassa:n, 1994: 116); z.B.
A: أنوجع Es tut mir weh. Aussagesatz
B: مع Warum?/Was tut dir weh?

Aber der Ausruf fordert den Hörer nicht auf, eine Frage zu stellen, sondern verlangt vielmals eine schnelle Reaktion: 

A: أوها oh!
B: * لماذ/إ: Warum?
Ausrufesatz

Das zeigt sich auch in dem Unterschied zwischen:

1. شّatta:n zaid wa amr! (Wie verschieden Zaid und 'Amr sind! / Welch ein großer Unterschied besteht doch zwischen Zaid und 'Amr!)

VS.

2. افترق زيد وعمرو. iftaraqa zaid wáamr. (Zaid und 'Amr haben sich getrennt.)

Satz (1) ist ein Ausrufesatz mit der Verwendung der Interjektion (شتان) (wie verschieden!/ Welch ein Unterschied!). Satz (2) dagegen ist ein Aussagesatz, der die Bedeutung des Satzes (1) weder wiedergeben noch beschreiben kann (vgl. Hassa:n, 1994: 116)

$\mathrm{Zu}$ den Ausrufen gehören nach Hassa:n die folgenden Kategorien (vgl. Hassa:n, 2000: 137):

\begin{tabular}{llll} 
& Schwur & Verträge \\
\hline عقد & Erstaunen & Interjektion des Verbs \\
حكاية صوت أخالة & Onomatopoetikum & Lob oder Tadel
\end{tabular}

واو القسم Schwurpartikel "wa:w" besteht, z.B.: و و wa(a)lla:hi (Bei Alla:h!).

العقود 'aluqu:d (die Verträge), die sich als offizielle oder mündlich formulierte Bekundungen der Akzeptanz oder Einwilligung darstellen, z.B. die Einwilligungssformeln bei einer Eheschließung:

زوجنى موكلتك zawiğni: muwakilatak (Ich bitte Sie um die Heirat von der Ihnen bevollmächtigten Braut!)

قوبلت زو اجها منك qabiltu zawa:ğiha: $\operatorname{mink}^{a}$ (Ich nehme es an!).

التعجب 'attáă̌ub (das Erstaunen/ die Verwunderung). Hassa:n bezeichnet sie als Ausrufesätze. Man kann das Gefühl des Erstaunens bzw. der Verwunderung mit 


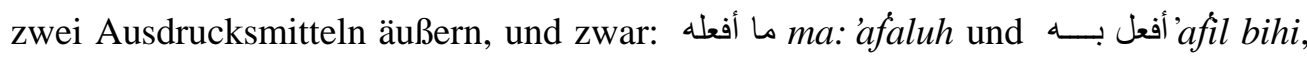
z.B.

ما أحسن زيداً! ma 'ahsana Zaid ${ }^{\text {an }}$ (Zaid ist ja gut!/ Wie gut ist doch Zaid!)

! 'ağmil bi-Muḥammad (Muḥammad ist ja schön!/ Wie schön ist doch Muḥammad!).

المدح والأم (Lob und Tadel): نعم ويئس 'ma und bi 'isa sind Ausdrücke, die Lob oder Tadel markieren, um die Billigung oder Missbilligung des Sprechers auszudrücken.

ni 'ma rrağulu Zaidun (Was für ein trefflicher Mann ist Zaid!.)

بئس خلقاً النفاق! bi sa huliqan annifaq ${ }^{\prime}$ (Welch schlechter Charakterzug ist die Heuchelei!).

الإخالة' al 'iha:la ${ }^{t u}$ (die Interjektionen der Verben bzw. Nomen der Verben), die die meisten Grammatiker "Nomen der Verben" nennen, z.B. هات Ha:t (Gib her!/bring her!/Her damit!, هيهات Hayha:t (Aber ach!).

! صِ șah (pst!)

هيهات بين هذا وذاك! hayha:t bayna had da: woda:k (welch ein Unterschied ist zwischen ihnen!) (Wehr, 2008: unter هيهات)

هَلا (Hikayatu(a)ssawti) Onomatopoetika, die Laute nachahmen, z.B. حكاية الصوت hala: (hey) für das Pferd, كَ kah und/oder kih ( pfui / lass das!) Ausruf des Ekels für das Kind, قب qab Klang des Fallens eines Schwerts.

Im Folgenden beziehe ich mich auf die Kategorien der Ausrufesätze von Hassa:n, denn ich möchte anmerken, dass die folgende Faktoren in Hassa:ns Untersuchung unberücksichtigt bleiben:

a) Die Einteilung der Ausrufe ist nicht strukturiert. In seiner Veröffentlichung im Jahr 1994 erwähnt Ḥassa:n: 


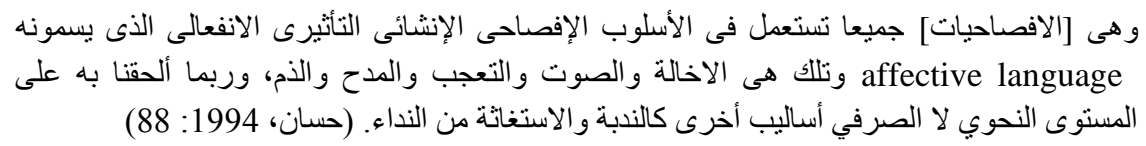

[Ü: [Die Ausrufe- bzw. Exklamativsätze] werden im Rahmen des affektiven emotionalen Ausrufs benutzt, der affektive Sprache genannt wird. Diese sind die Interjektion der Verben, die Onomatopoetika, Staunen und Verwunderung, Lob und Tadel; und vielleicht könnten wir dazu auch auf der syntaktischen und nicht auf der morphologischen Ebene andere Formeln wie Beklagensformeln und Hilferufe hinzufügen.] (Hassa:n, 1994: 88)

In demselben Werk verwendet Hassa:n - außer den oben genannten Kategorien auch andere Formen wie التحذير Warnung und الإغراء Anreiz aber nur auf syntaktischer und nicht auf morphologischer Ebene (vgl. Hassa:n, 1994: 117). Es ist merkwürdig, dass er diese wichtigen Arten übersieht.

b) Einige der von ihm genannten Arten des Ausrufesatzes bringen keine Empfindungen zum Ausdruck, wie die Onomatopoetika, die Verträge und der Schwur:

- Die Onomatopoetika stellen im Arabischen keine Ausrufesätze dar, sondern sind Ausdrücke des Ausrufs, denn sie erfüllen die Bedeutung des Ausrufs nur auf morphologischer und nicht auf syntaktischer Ebene ${ }^{6}$ (vgl. Ka:dah, 2005: 38).

- Die Verträge sind offiziell fixierte Bekundungen wie z.B. بعتكه bi tukah (Hiermit verkaufe ich es dir!). Sie wurden früher als Äußerungen des nicht auffordernden Ausrufs (bzw. der Exklamation) betrachtet, da sie keinen wahren oder falschen Sachverhalt feststellen und keine Forderung stellen. Semantisch transportieren sie überhaupt kein Gefühl oder keine emotionale Einstellung (vgl. Ka:dah, 2005: 38).

- In Bezug auf القسم (Schwur) führt Hassa:n einige Schwurformeln an: (ich schwöre bei Gott) (vgl. Hassa:n, 2000: 148). Diese Ausdrücke sind keine vollständigen Sätze, denn sie brauchen noch eine Aussage. Sie sind deshalb keine Ausrufesätze und äußern darüber hinaus keine Emotionen (vgl. Ka:dah, 2005: 39). 
c) Einige Formeln sind - meines Erachtens - den Ausrufesätzen hinzuzufügen, wie الندبة 'alnnudbatu (Beklagensformeln), die die Emotionen und Gefühl des Bedauerns und des Mitleids ausdrücken, z.B.: وازيداه wa:zaida:h (O Zaid!, Ach Zaid!, Ach du armer Zaid!) وارأساه warảsah (Ah/Ach mein Kopf!) (vgl. Hassa:n, 2000: 148).

Aus den oben genannten Gründen sollten - meines Erachtens - die Onomatopoetika, die Verträge und der Schwur aus den Ausrufesätzen entfernt und die Formeln von Beklagen, Warnen und Anreiz zu den Ausrufesätzen im Arabischen gezählt werden.

Die Verfasserin der Arbeit schließt sich an die Meinung von Mạ̣mu:d Aḥmad Naḥlah (1990) an, der den Terminus الانفعال (emotional) statt الإفصاح (expressiv) benutzt. Er benutzt statt الإنشاء غير الطلبي (wörtl. nicht aufforderender Ausruf) die Bezeichnung الإنشاء الانفعالي (wörtl. emotionaler Ausruf), die Wunsch, Hoffnung, Erstaunen, Lob und Tadel umfasst (vgl. Naḥlah, 19990: 7). Ich ziehe es vor, die Bezeichnung الجمل الإنشائية الانفعالية von Nahla beizubehalten.

\section{Der Vergleich}

Im Folgenden möchte ich auf den Aspekt näher eingehen, dass die Ausrufesätze im Deutschen und im Arabischen in vielen Fällen identisch sind:

1) Im Deutschen ,bringen [Ausrufesätze] die emotionale Beteiligung des Sprechers am dargestellten Sachverhalt zum Ausdruck.“" (Dürscheid, 2012: 64). Im Arabischen offenbaren und signalisieren die الجمل الإنشائية الانفعالية Ausrufesätze die emotionale Einstellung des Sprechers und zeigen die emotionalen Empfindungen.

Wandri:s bestätigt, dass die Ausrufe das Schreien aus schwerem Schmerz, aus plötzlicher Freude oder aus irgendeinem Gefühl heraus beinhalten; z.B. "آه! المسكين" „a:h! Almiski:n!““ (ach! der arme Mann!) bedeutet „,ich beklage jemanden’ oder "'أنت! ,' 'inta hina:’, (oh! Du bist ja hier!). Mit diesem Satz drückt der Sprecher sein Erstaunen aus, wenn er jemandem zufällig begegnet, ohne dass er erwartet hat (vgl. Wandri:s, 1950: 183). 
"فهذه الجمل ذات قيمة انفعالية واضحة كل الوضوح (...) تتفجر تلقائيا من النفس تحت نأثير انفعال شديد"

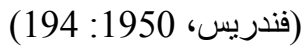

[Ü: Diese Sätze charakterisieren sich durch einen sehr klaren emotionalen Wert (...) sie werden automatisch aus der Seele unter dem Einfluss von extremer Emotion geäußert] (Wandri:s, 1950: 184, 194).

2) In beiden Sprachen steht die Emotionalität in den Ausrufesätzen im Vordergrund: Im Deutschen werden die Gefühle einer ,gewissen Emotionalität“" hervorgehoben (vgl. Rosengren, 1992: 264), z.B. „Wie herrlich leuchtet mir die Natur!“ (Goethe, Mailied). Im Arabischen kommt diese Satzart vor allem in Bereich der Stilistik, der Lyrik, sowie in Briefen und Tagebüchern vor (vgl. Hassa:n, 1994: 363).

3) Wie im Deutschen unterscheiden sich Aussagesätze und Ausrufesätze im Arabischen voneinander, auch wenn sie wie Aussagesätze aussehen.

Beispiele im Deutschen?

\section{Aussagesatz}

Der Baum ist sehr hoch.

Du bist heute sehr schön.

\section{Ausrufesatz}

Was für ein hoher Baum!

Gott, wie schön du heute bist!

Tabelle 2 Strukturunterschied zwischen Aussagesätzen und Ausrufesätzen im Deutschen. 
Beispiele im Arabischen:

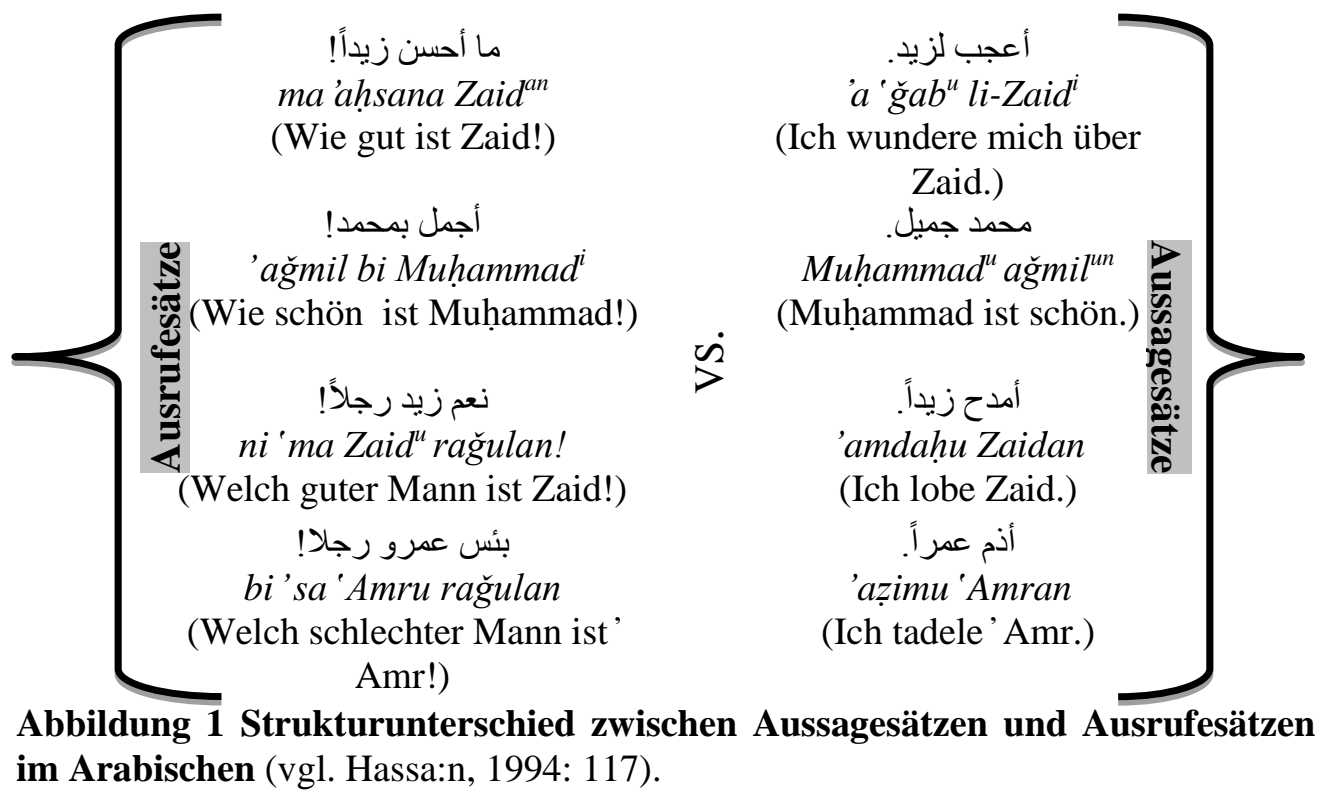

4) Diese Art von Sätzen werden gleichzeitig während des Geschehens ausgeäußert: Im Deutschen ruft der Sprecher etwas aus, was die gleiche Botschaft trägt. Im Arabischen bezeichnet sich diese Satzart ebenfalls als eine „Äußerung, die gleichzeitig zu dem stattfindet, worauf sie sich bezieht.“ (Stock, 2004: 15); z.B. Ist das hier eine Kälte!

5) In beiden Sprachen ist es möglich, dass niemand da ist, der diese Ausrufe hören oder danach fragen könnte; im Deutschen müssen sich die Ausrufe nicht unbedingt an einen Gesprächspartner richten. Ausrufe kann man "لا يتحتم فى هذه الحالة أن  unbedingt absichtlich] (Hassa:n, 1994: 363).

6) Im Deutschen bringen die Ausrufesätze Staunen, Verwunderung, Überraschung, Bewunderung, Bedauern oder Verärgerung zum Ausdruck. Im Arabischen kann der Ausrufesatz ein Ausdruck des Erstaunens bzw. der 
Verwunderung, des Lobs und Tadels, des Bedauerns oder des Wunsches sein.

7) Man darf auch nicht unerwähnt lassen, dass eine Beziehung zwischen den Interjektionen (Ausrufewörtern) und Ausrufesätzen besteht, denn die Interjektionen in beiden Sprachen signalisieren ebenfalls die emotionale Einstellung des Sprechers. Dazu liegen folgende Beweise vor; einerseits sind die Ausrufesätze durch die Interjektionen charakterisiert. Andererseits beschränken sich diese Sätze in vielen Fällen nur auf die Interjektionen wie beispielsweise: Ah! Aha! O! Ach! Wehe! Huh! Ei! Au! Pfui! Hurra! He! Na! Juhu! Super! Toll! (vgl. Rosengren, 1992: 303). Das hat Becker konstatiert, indem er äußert:

In der rhetorischen Terminologie entspricht der Interjektion die exclamatio. Hinsichtlich des für beide Konzepte konstitutiven Aspekts des Ausrufs besteht in der Forschung weitestgehende Einigkeit, dass er mit „Emotionsausdruck“ einhergeht. (Becker, 2016: 111).

Beachtenswert, dass während die Ausrufesätze sich auf die Äußerung des Erstaunens des Sprechers über etwas Unerwartetes beschränken, drücken die Interjektionen verschiedene spezifische Empfindungen aus (vgl. Fries, 1992: 329). Die deutsche Interjektion ach und ihre äquivalente Interjektion $\dot{\tau}^{\prime}$ 'a $\underline{h}$ im Arabischen können Ausrufe von Bedauern, Kummer und Überraschung sein. Darüber hinaus sind diese weitverbreiteten Interjektionen je nach Tonmuster und Gesprächssituation ein Ausdruck von Schmerz, Mitleid, Verlangen, plötzlichem Verstehen, Verwunderung und Erstaunen (vgl. Duden-Grammatik, 1995: 374, vgl. DudenDeutsches Universalwörterbuch, 2007: 106, vgl. Ibn Manẓu:r, 2003: 35).

8) Das Ausrufezeichen folgt meistens den Ausrufesätze in beiden Sprachen.

Daraus lässt sich die Schlussfolgerung ziehen, dass die Ausrufesätze durch die emotionale Funktion und die sprachliche Expressivität in beiden Sprachen augezeichnet sind. Es ist wichtig zu betonen, dass die Ausrufesätze im Deutschen 
die emotionale Einstellung des Sprechers zum Ausdruck bringen. Daher stellen diese Sätze die Gefühle von Erstaunen, Verwunderung, Bewunderung und auch andere Empfindungen dar. $\mathrm{Zu}$ dieser Frage im Arabischen besteht noch keine Einigung. Ich unterstütze gänzlich aber die Meinung von Naḥlah, dass die Ausrufesätze sich im Arabischen auf Erstaunen, Verwunderung, Lob, Tadel, Wunsch und Hoffnung beschränken (vgl. Naḥlah, 19990: 7).

Die Frage, die durch diesen Beitrag erklärt wurde, ist nach den Formen der Ausrufesätze in beiden Sprachen. Im Deutschen zeigt Rosengren die Formen und Strukturen solcher Sätze und fasst sie darin zusammen: „Verb-erst, Verb-zweit, Verb-letzt-Sätze und Sätze, eingeleitet durch eine w-Phrase" (Rosengren, 1992: 264). Im Arabischen haben diese Sätze ihre bestimmten Formen, die sich von den Strukturen der Aussage-, Aufforderungs- und Wunschsätze unterscheiden.

Man kann also zum Schluss kommen, dass das Deutsche und das Arabische fast in Übereinstimmung mit dem Thema „Ausrufesätze" stehen.

\section{Anmerkungen}

${ }^{1}$ Die Beispiele jeder Satzart stammen aus Dürscheid (2012: 62-64).

${ }^{2}$ Diese Beispiele stammen aus Rosengren (1992: 266).

${ }^{3}$ Diese Beispiele entnahmen wir aus „Exklamativsatz“ von d'Avis, Franz (2013): In: Meibauer, Jörg / Steinbach, Markus / Altmann, Hans: Satztypen des Deutschen.

${ }^{4}$ Im Deutschen weist das Ausrufezeichen auf die Ausrufe hin. Im Arabischen aber ist das Ausrufezeichen nicht das einzige Merkmal der Ausrufesätze, deshalb ist kein Problem, wenn das Ausrufezeichen den arabischen Ausrufesätzen nicht folgt. Aber die Wiedergabe dieser arabischen Ausrufesätze ins Deutsche sollte - meines Erachtens - mit dem Hauptzeichen dieser Sätze und zwar mit dem Ausrufezeichen beendet werden.

${ }^{5}$ Anmerkung: die Übersetzung der arabischen Zitate stammt von der Verfasserin der Arbeit und ist durch eckige Klammer gekennzeichnet.

${ }^{6}$ Ich bin der Meinung, die besagt, dass die Onomatopoetika - sowohl im Deutschen als auch im Arabischen - eine der Kategorien der Interjektionen sind und nicht als Ausrufesätze 
bezeichnet werden (vgl. Hassa:n, 1994: 116). Der Unterschied zwischen der Interjektion und dem Exklamativsatz ist wie der Unterschied zwischen Lexem und Satz, d.h. „Sie [Interjektion] kann deshalb auch allein auftreten und ist nicht von einem an sie gekoppelten Satztyp abhängig." (Rosengren, 1992: 303).

${ }^{7}$ Diese Beispiele wurden aus dieser Webseite http://www.gutefrage.net/frage/unterschiedzwischen-aussagesatz-und-ausrufesatz, am 10/10/2016 entnommen. 


\section{Literaturverzeichnis}

- Becker, Lisa (2016): Sprache und Rhetorik der Emotion im Partnerwerbungsgespräch. Tübingen. Narr Francke Attempto.

- Bussmann, Hadumod (Hrsg.) (2002): Lexikon der Sprachwissenschaft. 3. Aufl. Stuttgart.

- d'Avis, Franz (2013): Exklamativsatz. In: Meibauer, Jörg / Steinbach, Markus / Altmann, Hans: Satztypen des Deutschen. De Gruyter Lexikon, S. 171- 197.

- Duden (2007): Duden Universalwörterbuch.

- Duden (1995): Grammatik der deutschen Gegenwartssprache. Mannheim, Leipzig, Wien, Zürich.

- Dürscheid, Christa (2012): Syntax. Grundlagen und Theorien. 6. Aufl. Göttingen: Vandenhoeck \& Ruprecht.

- Kessel, Katja/ Reimann, Sandra (2012): Basiswissen Deutsche Gegenwartssprache, 4. durchges. Aufl. Narr Francke Attempto. Tübingen.

- Kürschner, Wilfried (1989): Grammatisches Kompendium. 3. Aufl. A. Francke Verlag. Tübingen, Basel.

- Rosengren, Inger (1992): Zur Grammatik und Pragmatik der Exklamation. In: Rosengren, Inger (Hrsg.): Satz und Illokution. Bd. 1. Niemeyer. Tübingen, S. 263-306.

- Stock, Kristina (2004): Arabische Stilistik. Dr. Ludwig Reichert Verlag. Wiesbaden.

- Wehr, Hans (2008): Arabisches Wörterbuch für die Schriftsprache der Gegenwart. 5. Aufl. Lorenz Kropfitsch. 


\section{Arabische Literatur}

$$
\text { ابن منظور (2003): لسان العرب، دار المعارف، القاهرة. }
$$

Ibn Manzu:r (2003): Lisa:nu('a)l'arab [Sprache der Araber], Da:ru('a)lma 'a:rif. Kairo.

$$
\text { • تمام حسان (1994): اللغة العربية معناها ومبناها: دار الثقافة }
$$

Hassa:n (1994): Allugatu('a)l arabiyyah Ma na:ha: wamabna:ha: [Die arabische Sprache, ihre Bedeutung und ihre Bildung]. Dar('a)ttaqa:fah.

$$
\text { • تمام حسان (2000): الخلاصة النحوية: عالم الكثب. }
$$

Hassa:n (2000): Alhula:șatu('a)nnahawiyyah. [Das syntaktische Fazit]:

'a:lamu('a)lkutib.

ج فندريس (1950): اللغة. تعريب: عبد الحميد الدواخلي ـ محمد القصاص .مكتبة الأنكلو المصرية

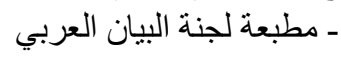

Wandri:s (1950): Allugah. [Die Sprache], Übersetzung ins Arabische 'abdelhami:d Eldawahli: - Muhammad Elkașșa:ș.

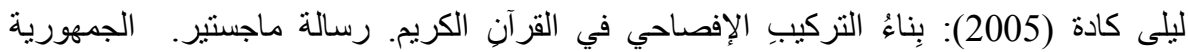

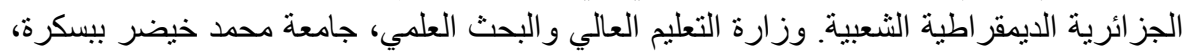

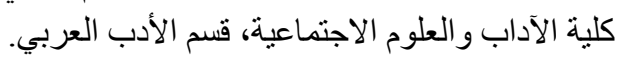

Ka:dah (2005): Bina:'u('a)ttarki:b Al'ifșa:hi: fi(:'a)lqura:ni('a)lkari:m [Zur Bildung der Ausrufekonstruktion im Koran]. Magisterarbeit. Algerische Demokratische Volksrepublik. Das Ministerium für Hochschulbildung und wissenschaftliche Forschung, Universität Muhammad Khider in Baskrah, Fakultät für Geistes- und Sozialwissenschaften an der Abteilung für arabische Literatur.

$$
\text { محمود أحمد نحلة (1990): علم المعاني، ـ دار العلوم العربيةـ بيروت_ لبنان }
$$

Nahlah (1990): 'ilmu('a)lma'a:ni: [Die semantische Wissenschaft]. Da:ru('a)l'ulu:mi('a)l'arabiyyah.

\section{Weblinks:}

- http://www.gutefrage.net/frage/unterschied-zwischen-aussagesatz-undausrufesatz, abgerufen am 10/10/2016.

- http://www.karstenrinas.com/pdf/Rinas-Partikeln als Mumien.pdf, abgerufen am 20/10/2016. 